\title{
Deadlock Avoidance of a Kind of JSP with Multi-resources Sharing ${ }^{*}$
}

\author{
Jing Li, Hejiao Huang, Farooq Ahmad \\ Dept. of Computer Science and Technology, Harbin Institute of Technology Shenzhen \\ Graduate School, China
}

\{hjhuang@hitsz.edu.cn; lijing@hitsz.edu.cn; farooq190@ hotmail.com\}

\begin{abstract}
This paper presents the scheduling problem with multi-resource sharing, which each operation may need more than one kinds of resource. Timed Petri net is used to formulate this problem to analyze deadlock and minimize the makespan. A deadlock avoidance policy addressed here consists of three stages: The first stage is deadlock detection based on transitive matrix and circular waiting. The second one is deadlock recovery by adding new arcs to destroy deadlock. The last stage is deadlock-free design. The efficiency of the method proposed in this paper is illustrated by an example in the end.
\end{abstract}

Keywords: Petri net, JSP, Deadlock Avoidance, Transitive matrix

\section{Introduction}

Job-shop scheduling problems (JSP) can be stated as follows: there are a finite set of $n$ jobs, each job which consists of a chain of operations, and a finite set of $m$ machines and some other resources, such as workers and AGVs, which are able to process at most one operation at a time. Each operation requires one or more than one resource, but at most one unit of each type of resource. Each resource can process at most one operation at a time, and each operation must be processed during an uninterrupted time on some given resources. The purpose is to find a schedule, that is, a reasonable allocation of the operations to time intervals to machines or other resources such that the makespan is minimal. The allocation of resources has to be considered for the modeling and functioning of JSP systems, which is a challenge for a system designer, especially in multi-resource sharing system.

Petri nets have been extensively used to model and analyze discrete event systems including JSP [1]-[4]. It is useful for preventing and avoiding unexpected system behaviors, such as deadlock and capacity overflow. Siphon are mainly technology on deadlock analysis [3]-[4]. Some scheduling algorithms are also used to get a feasible scheduling, such as heuristic scheduling algorithm and dynamic programming approach [5]-[6].

Transitive matrix of Petri net is another strategy to analyze deadlock [7]. The entries of place transitive matrix describe the transferring relation from one place to another place through transitions. Deadlock structures, deadlock avoidance method are given in [8]-[9]. The theorems above have been extensively studied to

* This work was financially supported by National Natural Science Foundation of China with Grant No. 10701030.

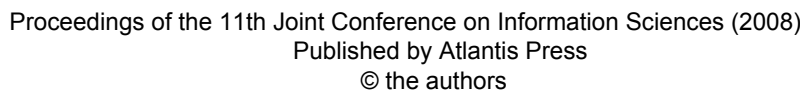


prevent deadlock and analyze the properties of Petri nets. However, the compositional system that shares common resources could not grab much attention of the researchers, especially for multiresources sharing system.

In this paper, timed Petri net is addressed to model the JSP with multiresources sharing. Deadlock conditions are analyzed by place transitive matrix and a deadlock recovery method is introduced to construct a deadlock-free system.

This paper is organized as follow. The next section introduces system modeling by timed Petri net. In Section 3, some basic knowledge about deadlock is presented. Deadlock avoidance is stated in Section 4 based on deadlock detection and recovery. Section 5 describes a case study about JSP. Some conclusive remarks are presented in section 6 .

\section{Timed Petri Net Modeling}

The notations used in this paper are mostly taken from [1], [9]-[10].

Definition 1 A Timed Petri net $(T P N)$ is a 6-tuple $\left(N, M_{0}\right)=\left(P, T, I, O, M_{0}, \tau\right)$, where $P$ is a finite set of places; $T$ is a finite set of transitions and $P \cap T=\varnothing$ and $P \cup T \neq \varnothing ; I: T \rightarrow P^{\infty}$ is the input function, a set of directed arcs from places to transitions, $I\left(t_{j}\right)$ is the set of input place of $t_{j}$; $O: T \rightarrow P^{\infty}$ is the output function, a set of directed arcs from transitions to places, $O$ $\left(t_{j}\right)$ is the set of output place of $t_{j} ; M_{0}$ is the initial marking. $\tau$ is a time function $\tau$ : $T \rightarrow N$ ( $N$ is the set of positive integers), which is associated to transitions such that $\forall t \in T, \tau(t)=n$ represents the firing time of transition $t$.

Definition $2 \forall p_{i} \in P$ and $\forall t_{j} \in T$, A place $p_{i}$ is an input place of transition $t_{j}$ if $p_{i} \in I\left(t_{j}\right)$ and $p_{i}$ is an output place of transition $t_{j}$, if $p_{i} \in O\left(t_{j}\right)$. Denote the weight of directed arc $\left(p_{i}, t_{j}\right)$ as \# $\left(p_{i}, I\left(t_{j}\right)\right)$, and the weight of directed arc of $\left(t_{j}, p_{i}\right)$ as \# $\left(p_{i}, O\left(t_{j}\right)\right)$.
Definition $3 B^{-}[i, j]$ is the matrix of input function with $m$ rows and $n$ columns, where $B^{-}[i, j]=\#\left(p_{i}, I\left(t_{j}\right)\right) ; B^{+}[i, j]$ is the matrix of output function with $m$ rows and $n$ columns, where $B^{+}[i, j]=\#\left(p_{i}, O\left(t_{j}\right)\right)$. $B=B^{+}-B^{-}$is called incidence matrix.

Definition 4 Let $L_{P}$ be place transitive matrix and let $L_{V}$ be transition transitive matrix with $m$ rows and $m$ columns, where $L_{P}=B^{-}\left(B^{+}\right)^{T}$ and $L_{V}=\left(B^{+}\right)^{T} B^{-}$. The labeled place transitive matrix $L_{V P}$ is a $m$ $\times m$ matrix satisfying: $L_{V P}=B^{-} \operatorname{diag}\left(t_{l}\right.$, $\left.t_{2}, \ldots, t_{n}\right)\left(B^{+}\right)^{T}$, where the elements of $L_{V P}$ describe the direct transferring relation from one place to another through one or more transitions. The Weighted place transitive matrix is denoted as $L_{V P}{ }^{*}$, If a transition $t_{k}$ appears $s$ times in the same column of $L_{V P}$, then replace $t_{k}$ in $L_{V P}$ by $t_{k} / s$ in $L_{V P}{ }^{*}$, otherwise the elements in $L_{V P}{ }^{*}$ are the same as $L_{V P}$.

Definition 5 Consider two Petri nets $\left(N_{1}\right.$, $\left.M_{10}\right)=\left(P_{1}, T_{1}, I_{1}, O_{1}, M_{10}\right)$ and $\left(N_{2}, M_{20}\right)$ $=\left(P_{2}, T_{2}, I_{2}, O_{2}, M_{20}\right)$, where $P_{1} \cap P_{2}=R$ $\neq \varnothing$. Let $\left(N, M_{0}\right)=\left(P, T, I, O, M_{0}\right)$ be composed from $\left(N_{1}, M_{10}\right)$ and $\left(N_{2}, M_{20}\right)$ by operation place fusion. Then the elements in $\left(N, M_{0}\right)$ are defined as follows: $P$ $=P_{1} \cup P_{2}, T=T_{1} \cup T_{2}, I=I_{1} \cup I_{2}, O=$ $\mathrm{O}_{1} \cup \mathrm{O}_{2}$ and

$$
M_{0}(p)=\left\{\begin{array}{cc}
M_{10}(p) & p \in P_{1} \\
M_{20}(p) & p \in P_{2} \\
\max \left\{M_{10}(p), M_{20}(p)\right. & p \in R
\end{array}\right.
$$

Each job will be a small Petri net with a unique path of transitions and places. Each operation in a job corresponds to a transition $t$, and the input (output) place of transition $t$ is an operation place $P$ that can be interpreted as the start (end) of the operation. The resource places are connected to the transitions which operations require these resources. Moreover, for each resource occupied by some operation $t$, when it is not used by

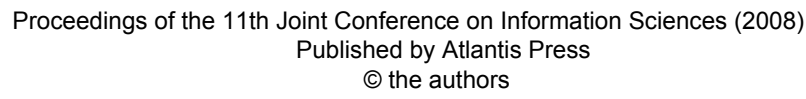


the next operation, this resource can be released after operation $t$ finished.

When the operations are ready to implement or the resources are available, there will be tokens in those places. Moreover, the original (final) state of each job corresponds to the initial marking $M_{0}$ (final marking $M_{f}$ ) of the Petri net model. The whole model is got by common place merging, and this $n$ jobs' scheduling problem is converted to find a firing sequence in the resultant Petri net model to get a deadlock-free system in order to calculate the makespan.

\section{Deadlock Analysis}

Some definitions about deadlock used in next section are presented here.

\subsection{Some Notations about Deadlock}

A transition is said to be a dead transition at marking $M$ if $M$ is not the final marking $M_{f}$ and there is no reachable marking to make transition $t$ enable. Formally, transition $t$ is a dead transition: $\exists M \in R\left(N, M_{0}\right) \wedge\left(M \neq M_{f}\right)$, $\forall t \in T:-M[t>$, then.

Since our modeling method is based on the composite designing, the "circular waiting" discussed in this paper is between two tasks. In manufacturing system, "circular waiting" is said to be a state that for two tasks, each of them hold a machine but both of them are waiting for the second machine occupied by the other. It is one condition of deadlock in manufacturing system.

In Petri net, "circular waiting" can be demonstrated as, for Petri net $N_{i}(i=1,2)$, $r_{1}$ and $r_{2}$ are resource places in $N_{i}$ and $t_{i a}$ $t_{i b} t_{i c} t_{i d}(i=1,2)$ is sub-transition path which is associated with resource places, where $N_{l}$ satisfies: $r_{l}$ is associated with the pairs of transitions $\left(t_{l a}, t_{l c}\right)$ and $\left(t_{l b}\right.$, $\left.t_{l d}\right)$, and $r_{l} \bullet=t_{l a}, \bullet r_{1}=t_{l c}$ and $r_{2} \bullet=t_{l b}$, $\bullet r_{2}=t_{l d} ; N_{2}$ satisfies: $r_{1}$ is associated with the pairs of transitions $\left(t_{2 a}, t_{2 c}\right)$ and $\left(t_{2 b}, t_{2 d}\right)$, and $r_{1} \bullet=t_{2 b}, \bullet r_{1}=t_{2 d}$ and $r_{2} \bullet=$ $t_{2 a}, \cdot r_{2}=t_{2 c}$.

Deadlock occurs when there are dead transitions that cause "circular waiting" in the system. A net is said to be deadlock-free if and only if there is no deadlock in the system.

\subsection{Transformation of Marking}

In this sub-section, the notation about transformation equation [7]-[9] and the improved form on JSP are presented here. The transformation of marking is defined as $M_{R}(k+1)^{T}=M(k)^{T} L_{V P}{ }^{*}$, where $M_{R}(k+1)$ is a $m$-vector of nonnegative integer and is a reachable marking from $M(k)=\left[p_{I}(k)\right.$, $\left.p_{2}(k), \ldots, p_{m}(k)\right]^{T}, L_{V P}{ }^{*}$ is the weighted place transitive matrix [9]. At a state $M(k)$, If $t_{i}$ fires, we define $\left|t_{i}\right|=1$; and if there is a token in $p_{i}$ at $M(k)$,define $\left|p_{i}(k)\right|=1$.

The transformation equation shows the flow relation of a token in $p_{i}(k)$ from $M(k)$ to $M_{R}(k+1)$ by the weighted place transitive matrix. In this equation, if $\sum_{i} p_{i} L_{V P_{i j}}{ }^{*}$ is integer, then let $\sum_{i} p_{i} L_{V P_{i j}}{ }^{*}=1$, which means $p_{i}(k)$ can be transferred from $M(k)$ to $M_{R}(k+1)$; otherwise, if $\sum_{i} p_{i} L_{V P_{i j}}{ }^{*}$ is not integer, let $\sum_{i} p_{i} L_{V P_{i j}}{ }^{*}=0$, which means $p_{i}(k)$ can not be transferred from $M(k)$ to $M_{R}(k+1)$ [9].

However, $M_{R}(k+1)$ only presents the flow relation of tokens, it may not necessary correspond to $M(k+1)$. In order to improve this situation, we add some constrained conditions to this equation:

(1) When $\sum_{i} p_{i} L_{V P_{i j}}{ }^{*}$ is integer, e.g. $\sum_{i} p_{i} L_{V P_{i j}}{ }^{*}=1$ in $M(k+1)$, then token can be transferred from $M(k)$ to $M(k+1)$;

(2) If $\sum p_{i} L_{V P_{i j}}{ }^{*}$ is not integer, $\sum p_{i} L_{V P_{i j}}{ }^{*}=0$ in $M(k+1)$, then token can't be transferred; 
(3) The token of place $p_{i}$ in condition (2) which is not the same as place in condition (1) is not changed;

(4) The token in other place is the same as $M_{R}(k+1)$.

From this calculation we can find out a series of reachable marking from $M_{0}$. In addition, if there is no reachable marking at $M_{i}$, the next reachable marking calculated will be the same as $M_{i}$. Thus we can find out whether a marking is reachable or not from $M_{0}$.

\section{Deadlock Avoidance Method}

Deadlock detecting algorithm is addressed to find out deadlock in the compositional system by merging two small Petri nets with shared resources.

\section{Algorithm I: Deadlock Detection}

Input: $N_{1}=\left(P_{I} \cup R, T_{1}, F_{1}, M_{10}, \tau_{l}\right), N_{2}=$ $\left(P_{2} \cup R, \quad T_{2}, \quad F_{2}, M_{20}, \tau_{2}\right)$, the Initial marking $M_{10}, M_{20}$ and the final marking $M_{l f}, M_{2 f}$.

Output: Deadlock or not

Step 1: Do place merging on $N_{i}$ and $N_{j}$, and then generate a net $N$ with the initial marking $M_{0}$ and the finial marking $M_{f}$.

Step 2: Construct the weighted place transitive matrix $-L_{V P}{ }^{*}$ for compositional net $N$.

Step 3: For $k=0, k<n, k++$

Calculate the next marking $M_{R}(k+1)$ from the marking $M_{R}(k)$ by $M_{R}(k+l)^{T}=$ $M(k)^{T} L_{V P}{ }^{*}$

If $M_{R}$ is equal to $M_{f}$, stop, output "There is no deadlock in the net, wecan get the makespan".

else if there are dead transitions and these dead transitions construct a "circular waiting" structure, then output "There is a deadlock, we should do deadlock recovery on the model”.

else output "There are mistakes in the system model”.

If deadlock occurs, the system falls into global deadlock and stops running. Therefore, deadlock recovery is important in system design. Since deadlock is caused by "circular waiting", if we destroy the "circular waiting" structure, deadlock will disappear and the system will be deadlock-free. Therefore, a recovery method is proposed using "selfloop" to destroy "circular waiting" structure in this subsection.

Consider, $N_{l}$ and $N_{2}$ are two Petri net models with shard places $r_{1}$ and $r_{2}$. They satisfy the "circular waiting" conditions mentioned in section 3.1. Suppose $\sigma_{i}=t_{i 0}$ $t_{i l} \ldots t_{i m}$ is a transition path in $N_{i}, \sigma_{i}{ }^{\prime}=t_{i a}$ $t_{i b} t_{i c} t_{i d}$ is a sub-transition path of $\sigma_{i}(i=$ $\{1,2\})$, and \# $\left(\begin{array}{llll}t_{i 0} & t_{i 1} & \ldots & t_{i(a-l)}\end{array}\right)$ is the makespan of $t_{i 0} t_{i 1} \ldots t_{i(a-l)}$.

Algorithm II: Deadlock Recovery

Input: Dead transitions which cause deadlock, suppose they are $t_{l b}$ and $t_{l b}$.

Output: A recovery model

Step 1: Compare \# $\left(t_{10} t_{11} \ldots t_{l(a-l)}\right)$ and \# $\left(\begin{array}{llll}t_{20} t_{21} & \ldots & t_{2(a-1)}\end{array}\right)$ and find out the greater quantity, without loss the generality, suppose it is \# $\left(t_{10} t_{11} \ldots t_{l(a-l)}\right)$.

Step 2: Add two new arcs $t_{l(b-l)} \rightarrow r_{l}$ and $r_{l} \rightarrow t_{l b}$ into the model, then the pair $\left(t_{l a}\right.$, $\left.t_{l c}\right)$ is decomposed as $\left(t_{l a}, t_{l(b-l)}\right)$ and $\left(t_{l b}\right.$, $t_{l c}$ ) which are both associating with $r_{l}$.

Step 3: Deadlock detection by algorithm I. If output is "There is a deadlock", do the recovery similarly as Step1 and Step2.

else, there is no deadlock, stop.

After the recovery given above, the sub-system becomes deadlock-free. The method proposed above only change the input and output of a resource place in one sub-Petri net. If the complexity of the model is not considered, we can do the similar change in the second sub-Petri net, and the Petri net model may be live and the makespan will be minimized at a time.

\subsection{Deadlock-free Method}

The deadlock detection and recovery algorithm are mainly for a compositional system with two small Petri nets sharing common resources. For an $n$ jobs Petri 
nets system, we do deadlock detection and recovery on each pair of Petri nets. Then do place merging on these $n$ Petri nets and a deadlock-free modeling is got. The makespan of the scheduling can be calculated by simulations on CPN-Tool.

\section{A Case Study}

An example with two jobs and two kinds of resources is shown in Table 2 in order to describe the technology of deadlock detection and recovery.

Table 2: A Job Shop Scheduling Problem

\begin{tabular}{|c|c|c|c|c|}
\hline & $\mathrm{O}_{1}$ & $\mathrm{O}_{2}$ & $\mathrm{O}_{3}$ & $\mathrm{O}_{4}$ \\
\hline $\mathrm{J}_{1}$ & $\mathrm{M}_{1}: 7$ & $\mathrm{M}_{1}, \mathrm{M}_{2}: 4$ & $\mathrm{M}_{1}, \mathrm{M}_{2}: 3$ & $\mathrm{M}_{2}: 4$ \\
\hline $\mathrm{J}_{2}$ & $\mathrm{M}_{2}: 3$ & $\mathrm{M}_{1}, \mathrm{M}_{2}: 5$ & $\mathrm{M}_{1}, \mathrm{M}_{2}: 4$ & $\mathrm{M}_{1}: 3$ \\
\hline
\end{tabular}

In order to get the scheduling, we solve this problem gradually:

Step 1: System model

Fig. 2 depicts the Petri nets for two jobs, and $t_{i 1}, t_{i 2}, t_{i 3}, t_{i 4}$ are corresponding to the four operations in job $i(i=\{1,2\})$, $r_{1}$ and $r_{2}$ are corresponding to the two resources. The initial marking of job $i$ is $M_{i 0}=(1,0,0,0,0,1,1)^{\mathrm{T}}$ and the final marking is $M_{i f}=(0,0,0,0,1,1,1)^{\mathrm{T}}$.
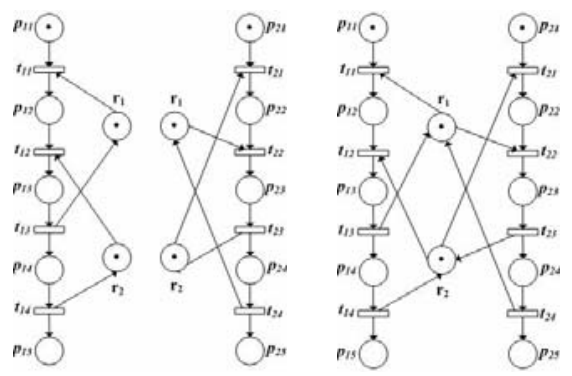

Fig.2. Petri nets models Fig.3. Place fusion

Step 2: Deadlock detection

- Do place merging on the common resources places $r_{1}$ and $r_{2}$ :

Fig. 3 is the compositional system for this problem, the initial marking of the merging system $M_{0}=(1,0,0,0,0,1,0,0$, $0,0,1,1)^{\mathrm{T}}$ and the final marking $M_{f}=(0$, $0,0,0,1,0,0,0,0,1,1,1)^{\mathrm{T}}$.
- Construct the weighted place transitive matrix $-L_{V P}{ }^{*}$ :

Table 3 is the weighted place transitive matrix of Fig.3, $M(k)=\left[p_{11}(k)\right.$, $p_{12}(k), p_{13}(k), p_{14}(k), p_{15}(k), p_{21}(k), p_{22}(k)$, $\left.p_{23}(k), p_{24}(k), p_{25}(k), r_{l}(k), r_{2}(k)\right]^{\mathrm{T}}$ and the initial marking is $M_{0}=(1,0,0,0,0,1,0$, $0,0,0,1,1)^{\mathrm{T}}$.

Table 3: $L_{V P}{ }^{*}$ of Petri net in Fig.3

\begin{tabular}{|c|c|c|c|c|c|c|c|c|c|c|}
\hline$p_{11}$ & $\begin{array}{ll}0 & t_{11} / 2\end{array}$ & 0 & 0 & 0 & 0 & 0 & 0 & 0 & 0 & \\
\hline$p_{12}$ & $\begin{array}{ll}0 & 0\end{array}$ & $t_{12} / 2$ & 0 & 0 & 0 & 0 & 0 & 0 & 0 & 0 \\
\hline$p_{13}$ & 0 & 0 & $t_{13}$ & 0 & 0 & 0 & 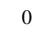 & 0 & 0 & $t_{13}$ \\
\hline$p_{14}$ & 0 & 0 & 0 & $t_{14}$ & 0 & 0 & 0 & 0 & 0 & 0 \\
\hline$p_{15}$ & 0 & 0 & 0 & 0 & 0 & 0 & 0 & 0 & 0 & 0 \\
\hline & 0 & 0 & 0 & 0 & 0 & $t_{21} / 2$ & 0 & 0 & 0 & 0 \\
\hline$p_{22}$ & 0 & 0 & 0 & 0 & 0 & 0 & $t_{22} / 2$ & 0 & 0 & 0 \\
\hline$p_{23}$ & 0 & 0 & 0 & 0 & 0 & 0 & 0 & $t_{23}$ & 0 & 0 \\
\hline$p_{24}$ & 0 & 0 & 0 & 0 & 0 & 0 & 0 & 0 & $t_{24}$ & $t_{24}$ \\
\hline$p_{25}$ & 0 & 0 & 0 & 0 & 0 & 0 & 0 & 0 & 0 & 0 \\
\hline$r_{1}$ & $\begin{array}{ll}0 & t_{11} / 2\end{array}$ & 0 & 0 & 0 & 0 & 0 & $t_{22} / 2$ & 0 & 0 & 0 \\
\hline$r_{2}$ & 0 & $t_{12} / 2$ & 0 & 0 & 0 & $t_{21} / 2$ & 0 & 0 & 0 & 0 \\
\hline
\end{tabular}

Calculate the reachable marking by transformation of marking: $M_{R}(k+1)^{T}=$ $M(k)^{T} L_{V P}{ }^{*}$ :

The next reachable marking is:

$M_{1}=M_{0} L_{V P}{ }^{*}=\left[0, p_{11}\left(t_{11} / 2\right)+r_{l}\left(t_{11} / 2\right)\right.$, $r_{2}\left(t_{12} / 2\right), 0,0,0, p_{21}\left(t_{2 l} / 2\right)+r_{2}\left(t_{21} / 2\right), r_{I}$ $\left.\left(t_{22} / 2\right), 0,0,0,0\right]^{\mathrm{T}}=[0,1,0,0,0,0,1,0$, $0,0,0,0]^{\mathrm{T}}$, then

$M_{2}=M_{1} L_{V P}{ }^{*}=\left[0,0, p_{12}\left(t_{12} / 2\right), 0,0,0,0\right.$, $\left.p_{22}\left(t_{22} / 2\right), 0,0,0,0\right]^{\mathrm{T}}=[0,0,0,0,0,0,0$, $0,0,0,0,0]^{\mathrm{T}}$

In $M_{2}$, transitions $t_{12}$ and $t_{22}$ are dead transitions connected to resource places $r_{l}$ and $r_{2}$, which involve "circular waiting”. Then the system is confronted a deadlock. Step 3: Deadlock recovery

- Since $\left(\# t_{11}=7\right)>\left(\# t_{21}=3\right)$, the first sub-model will be modified;

Add two arcs $t_{11} \rightarrow r_{1}$ and $r_{1} \rightarrow t_{12}$ to get a recovery model in Fig.4.;

- Deadlock detection again and there is no deadlock, stop.

When there are more than two jobs, deadlock detection and recovery should be implemented in each two jobs to make sure each sub-system deadlock-free. Then a deadlock-free compositional model can be built by place fusion. 


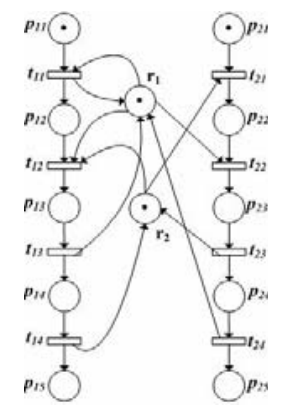

Fig.4.The recovery mode

Step 4: Scheduling resolution

Based on the algorithms above, the system is deadlock-free. The makespan of this JSP is 30, and the Gantt chart is shown in Fig. 5.

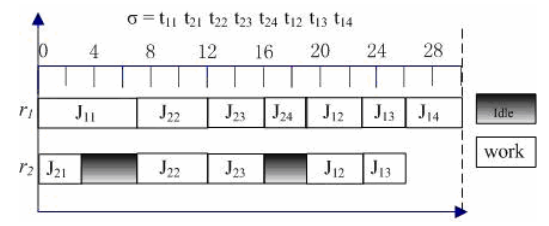

Fig.5. Gunter chart of the scheduling

\section{Conclusion}

In this paper, deadlock avoidance method in JSP with multi- resources sharing is considered by timed Petri nets. Place transitive matrix is applied to detect deadlock. A deadlock recovery strategy is introduced based on "self-loop". However, the work focuses on deadlock caused by two tasks. Our future work will do some deeply research in a more complex system, which deadlock is caused by more than three tasks with more kinds of shared resources.

\section{Reference}

[1] J. L. Peterson, "Petri Net Theory and the Modeling of System”, Prentice Hall, (1981).

[2] H.J. Huang, L. Jiao and T.Y. Cheung, "Property-preserving subnet reductions for designing manufacturing systems with shared resources", Theoretical Computer Science, 332(1-3): 461-485(2005).

[3] Hesuan $\mathrm{Hu}$, Zhiwu $\mathrm{Li}$ and Anrong Wang, "On the optimal set of elementary siphons in Petri nets for deadlock control in FMS”, 2006 IEEE International Conference of Networking, Sensing and Control, pp. 244-247(2006).

[4] Zhiwu Li and $\mathrm{Na}$ Wei, "Deadlock control of flexible manufacturing systems via invariant-controlled elementary siphons of Petri nets", The International Journal of Advanced Manufacturing Technology, Vol. 33, pp. 24-35(2007).

[5] Xu Gang and Wu Zhiming, “A kind of deadlock-free scheduling method based on Petri net”, IEEE HASR'02, pp. 195-200(2002).

[6] Kim Y., Tatsuya T., and Taysuo N., "FMS scheduling based on timed Petri net model and reactive graph search”, Applied Mathematical Modelling, Vol.31, pp.955-970(2007).

[7] Liu J., Itoh Y., Miyazawa I. and Sekiguchi T., "A research on Petri net properties using transitive matrix", Proceeding IEEE SMC99, pp. 888893(1999).

[8] Yujin. Song and Jongkun. LEE, "Deadlock analysis of Petri nets using the transitive matrix”, SICE Annual Conference 2002, Osaka, (2002).

[9] Sanghwan Kim, Sangho Lee and Jongkun Lee, "Deadlock analysis of Petri nets nased on the reousrce share places relationship”, IMACS multiconference on CESA, pp. 5964(2006).

[10] Murata T, "Petri net: properties, analysis, and applications”, Proc IEEE, Vol. 77, pp. 541-580(1985).

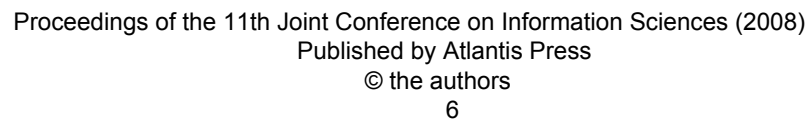

